\title{
Effects of allergen on airway narrowing dynamics as assessed by lung-slice technique
}

\author{
A.D. Chew, J.A. Hirota, R. Ellis, J. Wattie, M.D. Inman and L.J. Janssen
}

\begin{abstract}
Asthma is characterised by an excessive airway narrowing in response to a variety of stimuli, called airway hyperresponsiveness (AHR). Previous comparisons between mouse strains have shown that increased velocity of airway narrowing correlates with baseline airway responsiveness. These data prompted the investigation into models of induced AHR to see whether airway narrowing dynamics correlated with in vivo responsiveness.

In an attempt to reproduce some of the features of asthma, BALB/c mice were sensitised and subjected to either brief or chronic periods of allergen exposure. Brief exposure involved two challenges with intranasal chicken egg ovalbumin (OVAin). Chronic exposure involved six 2-day periods of OVAin challenges, each separated by 12 days. Control mice received intranasal saline challenges. Outcomes included videomicrometry of lung slices (magnitude and velocity of airway narrowing), in vivo respiratory physiology measurements and histological staining with morphometric analysis.

Neither brief nor chronic allergen exposure resulted in greater airway narrowing and increased velocity compared with saline controls. Structural changes in the airway, such as goblet cell hyperplasia, subepithelial fibrosis and increased contractile tissue, were detected in mice chronically challenged with allergen.

In conclusion, increased responsiveness to methacholine following allergen challenge may not be due to an intrinsic change to the smooth muscle per se, but rather to other changes in the lung, which ultimately manifest as an increase in respiratory resistance.
\end{abstract}

KEYWORDS: Airway smooth muscle, asthma, contraction, lung slices, remodelling

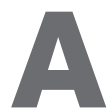
sthma is a chronic inflammatory lung disease characterised by variable airflow obstruction, airway inflammation and airway hyperresponsiveness (AHR). AHR refers to the increased ability of the airways to narrow following exposure to bronchoconstrictor agonists, such as methacholine and histamine, as compared with normal individuals [1]. The pathophysiological mechanisms that contribute to AHR are still unclear, although ongoing airway inflammation and airway remodelling are believed to play major roles, and elevated interleukin (IL)-13 levels seem to be necessary and sufficient for AHR in brief animal models of allergic asthma $[2,3]$. It has been suggested that these allergic mediators can act directly on airway smooth muscle (ASM) to increase contractile responses [4]. Airway remodelling describes the structural changes of airway walls observed in asthmatics, and includes ASM hypertrophy and/or hyperplasia, airway fibrosis and increased mucus production.
ASM contraction in vivo is neither isometric nor isotonic but rather auxotonic, in which the ASM shortens against an increasing load imposed by the attachments of the surrounding lung parenchyma, parallel elastic elements (i.e. extracellular matrix) and mucosal folding. Although it is known that isometric force measurements do not completely reproduce the in vivo situation, many studies use isometric force generation as the index of contractile function [5-9]. Many investigators have used isometric force generation as an index of AHR; however, isometric force measurements are currently thought to underestimate differences in airway responsiveness. Recently, airway contraction dynamics, namely maximal narrowing and velocity of narrowing, have been proposed to be more relevant markers of AHR, thus raising the possibility that airway dysfunction may arise from abnormalities within the smooth muscle itself. Previous studies have examined airway smooth muscle dynamics in naïve, nonallergen sensitised dogs [5], rats [10]

\section{AFFILIATIONS}

Firestone Institute for Respiratory Health, St Joseph's Healthcare, Dept of Medicine, McMaster University, Hamilton, ON, Canada.

\section{CORRESPONDENCE}

L.J. Janssen

St Joseph's Hospital

Room L314

50 Charlton Avenue East

Hamilton

Ontario

Canada L8N 4 A6

Fax: 19055406510

E-mail: janssenl@mcmaster.ca

Received:

June 282007

Accepted after revision:

November 062007

SUPPORT STATEMENT

The study was supported through an R\&D programme grant kindly

provided by the Canadian Institutes of Health Research

STATEMENT OF INTEREST

A statement of interest for this study can be found at www.eri.ersjournals.com/misc/ statements.shtml 
and mice $[6,7,11]$ but, to the present authors' knowledge, no other group has used a murine model of allergen-induced AHR to investigate airway contraction dynamics. Previous comparisons between naïve mouse strains have shown that increased velocity of airway narrowing correlates with airway responsiveness [6]. Consequently, the purpose of the present study was to examine the ASM-mediated airway narrowing dynamics and determine whether this could account for increased responsiveness to methacholine in vivo in a murine model of allergic asthma.

\section{MATERIALS AND METHODS \\ Animals}

Female BALB/C (wild type) mice were purchased (Harlan Sprague Dawley Inc., Indianapolis, IN, USA). Mice were aged 6-8 week (chronic protocol) or 10-12 week (brief protocol) and housed in environmentally controlled, specific pathogen-free conditions for 1 week prior to and for the duration of the experiments. All experimental procedures were approved by the Animal Research Ethics Board at McMaster University (Hamilton, ON, Canada), and conformed to the National Institute of Health guidelines for experimental use on animals.

\section{Sensitisation}

BALB/c mice were sensitised as described previously [12]. Briefly, mice received intraperitoneal (IP) injections of ovalbumin (OVA) with aluminum potassium sulfate on days 1 and 11, and with intranasal OVA (OVAin) on day 11.

\section{Exposure}

Sensitised BALB/C mice were subjected to either brief or chronic allergen exposure protocols. The brief protocol involved two 2 -day periods of OVAin (100 $\mu \mathrm{g}$ in $25 \mu \mathrm{L}$ saline) challenges. The chronic protocol involved six 2-day periods of OVAin, each separated by 12 days (total of 12 exposures over a 10 -week period). Control mice were subjected to the same sensitisation protocol but received intranasal saline exposures. Mice subjected to the brief exposure protocol were studied $24 \mathrm{~h}$ after the final exposure to either allergen or saline and chronic mice were studied 4 weeks after the final exposure to either allergen or saline. Separate groups of mice (10 saline control, 10 allergen-exposed) were studied in each protocol (brief versus control) and analysed using the thin lung-slice technique.

\section{Solutions}

Agarose type-VII solution (4\% (weight/volume); Sigma Chemical Corp., St Louis, MO, USA) was dissolved in distilled water at $60^{\circ} \mathrm{C}$, cooled to $37^{\circ} \mathrm{C}$ and mixed with $2 \times$ concentration Hanks' Balanced Salt Solution (HBSS) to give a $2 \%$ agaroseHBSS solution at $37^{\circ} \mathrm{C}$.

\section{Preparation of lung slices}

Lung slices were prepared as previously described in mice [13] with slight modifications. Briefly, mice were euthanised by $\mathrm{CO}_{2}$ followed by terminal exsanguination. The trachea was exposed and cannulated using a blunt-ended 19-gauge needle, followed by chest wall removal to expose the lungs. The lungs were inflated with $\sim 1.2 \mathrm{~mL} 2 \%$ agarose-HBSS solution at $37^{\circ} \mathrm{C}$. To clear the airway lumen, $0.1-0.2 \mathrm{~mL}$ of air was injected to flush the agarose-HBSS solution out of the airways into the alveolar tissue. The lungs were rinsed with $1 \times$ concentration HBSS at $4^{\circ} \mathrm{C}$ and the whole mouse preparation was placed at $4^{\circ} \mathrm{C}$ for $15 \mathrm{~min}$. The lungs were removed and placed in HBSS at $4{ }^{\circ} \mathrm{C}$ for an additional $30 \mathrm{~min}$ to ensure the complete gelling of the agarose within the lungs. The lungs were separated into individual lobes and bathed in cold HBSS. Using a tissue slicer (model EMS-4000; Electron Microscope Sciences, Fort Washington, PA, USA), $\sim 120 \mu \mathrm{m}$ thick slices from the right upper lobe were cut in $\mathrm{HBBS}$ at $4^{\circ} \mathrm{C}$.

\section{Incubation media for tissue slices}

Lung slices were transferred to Dulbecco's Modified Eagle's Medium (Invitrogen, Carlsbad, CA, USA) with $15 \mathrm{mM} \mathrm{4-(2-}$ hydroxyethyl)-1-piperazine ethanesulfonic acid buffer, L-glutamine and pyridoxine $\mathrm{HCl}$, supplemented with penicillinstreptomycin, amphotericin B, L-ascorbic acid, transferrin, selenium and insulin, and then incubated overnight at $37^{\circ} \mathrm{C}$.

\section{Image acquisition}

Lung slices were placed between two glass cover slips and held in position by a piece of nylon mesh (CMN-300-B; Small Parts, Miami Lakes, FL, USA) on the stage of the microscope (Nikon TMD, Nikon Canada Inc., Mississauga, ON, Canada) and viewed at $100 \times$ magnification. Lung slices were selected for study only if: 1) the airway of interest was free of agarose; 2) beating of cilia was observed; and 3) the epithelium of the airway was intact. Phase contrast images were recorded with a digital charge-coupled device camera (CV-252; Nikon), a frame-grabber board and image acquisition software (Video Savant; IO Industries, London, ON, USA). Frames were captured in time-lapse (except for data used to test the effect of frame rate, which was performed at a rate of 1 frame every $5 \mathrm{~s}$ ) for the allergen experimental protocols, stored in tagged image format (.tif) stacks of several hundred frames and analysed using Scion image analysis software (Scion Corporation, Frederick, MD, USA). In particular, images with 10-bit grey scale resolution were converted to binary by defining all pixels greater than a given brightness to white (e.g. the background seen through the lumen), and all those less intense to black (e.g. tissue). Additionally, all contiguous white pixels were identified as objects/particles, excluding any bounded object smaller than a given size (defined by the user to be much smaller than the airway lumen area, but much larger than any passing debris) and then the number of (white) pixels within any object larger than the cut-off value were counted. Given the dramatic difference in brightness between the airway lumen and any object in view (parenchymal/wall tissue or debris), minor changes in threshold level would have little or no effect on the absolute measurements made, and would have no effect whatsoever on the relative changes reported herein (i.e. the changes in diameter occurring from start to finish in a given recording).

\section{In vitro measurement of airway narrowing}

The lung slice was superfused for 5 min with $1 \times$ concentration HBSS in order to obtain a baseline. After baseline images had been recorded, a constant flow of $1 \mu \mathrm{M}$ acetylcholine was superfused over the lung-slice preparation for $5 \mathrm{~min}$. BERGNER and SANDERSON [13] have shown this concentration of acetylcholine to evoke nearly maximal responses. Airway area was measured with respect to time by pixel summing, using 
the Scion software (Scion Corperation). The cross-sectional airway area ranged $17,000-44,000 \mu^{2}$ (corresponding to a diameter range $145-235 \mu \mathrm{m})$. Airway narrowing was expressed as the percentage decrease in airway area in comparison to the initial airway area measurement. The peak velocity of airway narrowing was calculated using a simple mathematical algorithm (SigmaPlot; Systat Software Inc., Point Richmond, CA, USA), which numerically differentiates the values of area with respect to time (calculates the slopes between every point in the lumen area versus time plots).

\section{In vivo measurement of responsiveness to methacholine}

Respiratory physiology measurements were obtained using the flexiVent ${ }^{\mathrm{TM}}$ rodent ventilator system (Scientific Respiratory Equipment Inc., Montreal, Canada). Responsiveness to methacholine was measured on the basis of the response of total respiratory system resistance (RRS) to increasing intravenous doses of methacholine $(n=10$ per group). Each mouse was anaesthetised with Avertin (2,2,2-tribromoethanol; SigmaAldrich Canada Ltd., Oakville, ON, Canada) via IP injection at a dose of $240 \mathrm{mg} \cdot \mathrm{kg}^{-1}$ and underwent tracheostomy with a blunted 18-gauge needle, then were connected to the flexiVent ${ }^{\mathrm{TM}}$ computer-controlled small animal ventilator (Scientific Respiratory Equipment Inc.). Animals were ventilated quasisinusoidally (150 breaths $\cdot \mathrm{min}^{-1}, \quad 10 \mathrm{~mL} \cdot \mathrm{kg}^{-1}$, inspiratory/ expiratory time ratio of $66.7 \%$ and pressure limit of $30 \mathrm{cmH}_{2} \mathrm{O}$ ). A script for the automated collection of data was then initiated, with the positive end-expiratory pressure (PEEP) level set at $2 \mathrm{cmH}_{2} \mathrm{O}$ and default ventilation for mice. After the mouse was stabilised on the ventilator, the internal jugular was cannulated using a 25-gauge needle. Paralysis was achieved using pancuronium $\left(0.03 \mathrm{mg} \cdot \mathrm{kg}^{-1}\right.$ administered intravenously) to prevent respiratory effort during measurement. To provide a constant volume history, data collection was preceded by a 6-s total lung capacity perturbation (peak amplitude $25 \mathrm{cmH}_{2} \mathrm{O}$ ). After $20 \mathrm{~s}$, saline was injected intravenously followed by 10, 33, 100 and $330 \mu \mathrm{g} \cdot \mathrm{kg}^{-1}$ methacholine (ACIC, Brantford, ON, Canada). For each dose, a maximum of 13 "QuickSnap-150" perturbations (single sinusoidal inspiration/expiration ratio of $0.4 \mathrm{~s}$ duration with a volume amplitude relative to weight of $10 \mathrm{~mL} \cdot \mathrm{kg}^{-1}$ ) were performed for $\sim 45 \mathrm{~s}$, followed $10 \mathrm{~s}$ later by a 6 -s TLC. Each breath was delivered after allowing the mouse to expire passively for $1 \mathrm{~s}$ against a positive pressure of $2 \mathrm{cmH}_{2} \mathrm{O}$. Respiratory mechanics (RRS and elastance resistance (ERS)) were calculated from simultaneous recording of airway pressure, change in lung volume and airflow in or out of the lungs, using multiple linear regression to fit these data to the following linear single compartment model of the mouse respiratory system:

$$
P=(\text { ERS } \times V)+\left(\text { RRS } \times V^{\prime}\right)+P_{\mathrm{O}}
$$

where $P$ is pressure, $P_{0}$ is the end alveolar pressure determined by the PEEP applied to the expired line of the ventilator, $\mathrm{V}$ is volume and $V^{\prime}$ is flow. After the last dose was complete, the mouse was removed from the ventilator for further tissue collection. Cardiac frequency and oxygen saturation were monitored via infrared pulse oxymetry (Biox 3700; Ohmeda, Boulder, CO, USA) using a standard ear probe placed over the proximal portion of the mouse's hind limb.

\section{Lung histology and morphometry}

The lungs were processed as described in detail previously [14]. Following agar infusion, the left lobe was fixed in formalin for $24 \mathrm{~h}$, after which the left lung was cut in half and imbedded in paraffin. Transverse sections $(3-\mu \mathrm{m}$ thick) were cut and assessed with the following stains: periodic acid Schiff, Picro-Sirius red, and $\alpha$ - smooth muscle actin (SMA).

\section{Statistical analysis}

All data are expressed as mean \pm SEM and $n$ refers to the number of animals used. Only one airway from one lung slice was used from each animal. Statistical comparisons between groups were carried out using an unpaired t-test and p-values $<0.05$ were considered to be significant.

\section{RESULTS}

Brief or chronic allergen exposure increases methacholine responsiveness in $B A L B / c$ mice

In order to confirm that the allergen exposure protocol was associated with increased cholinergic responsiveness in mice, in vivo respiratory function was measured in BALB/c mice following brief or chronic allergen exposure (these mice were separated from the animals used for lung-slice experiments). These mice showed significant increases in maximal methacholine-induced RRS compared with corresponding saline controls $(n=10 ; p<0.05 ;$ fig. 1$)$. Identical observations were made in similarly treated mice, where respiratory responses to methacholine were measured using a flow interruptor method (data not shown) [12].

\section{Validation of experimental technique}

Several technical aspects of the lung-slice technique used herein have been validated: extent of inflation; concentration of agarose; and wide range of airway sizes [15]. In order to evaluate the optimal rate of data acquisition for estimating contraction velocities, a single recording at 10 frames per second (fps) was performed and divided into several other videos with lower frame rates by selecting appropriate frames (e.g. every 10th frame to obtain an effective video rate of $1 \mathrm{fps}$ ). The latter were then analysed for velocity of constriction.

Figure 2a shows a segment of the 10-fps recording of the constrictor response to acetylcholine and the estimations of instantaneous velocities made from a 120-s portion of that video (beginning $\sim 30 \mathrm{~s}$ prior to cholinergic stimulation) are given in the top tracing in figure $2 b$. The noise in the latter is primarily produced by minor changes in airway lumen area from one frame to the next as the muscle twitches, a phenomenon previously described by BERGNER and SANDERSON [13]. Other possible sources of noise $(<5 \%)$ may pertain to debris drifting through the field of view and/or subtle changes in illumination. This noise might hamper any accurate determination of peak velocity of airway closure.

Figure $2 \mathrm{~b}$ also shows the estimations of velocity obtained at an effective video rate of 5 fps (only every other frame included), as well as slower effective video rates down to $0.1 \mathrm{fps}$ (only every 100th frame). Decreasing the video rate to 1 or $0.5 \mathrm{fps}$ substantially reduces the inherent noise in the velocity estimations, revealing an underlying oscillation with a frequency of $\sim 0.33 \mathrm{~Hz}$, as well as the peak velocity of acetylcholine-evoked contraction per se. Reducing the effective 

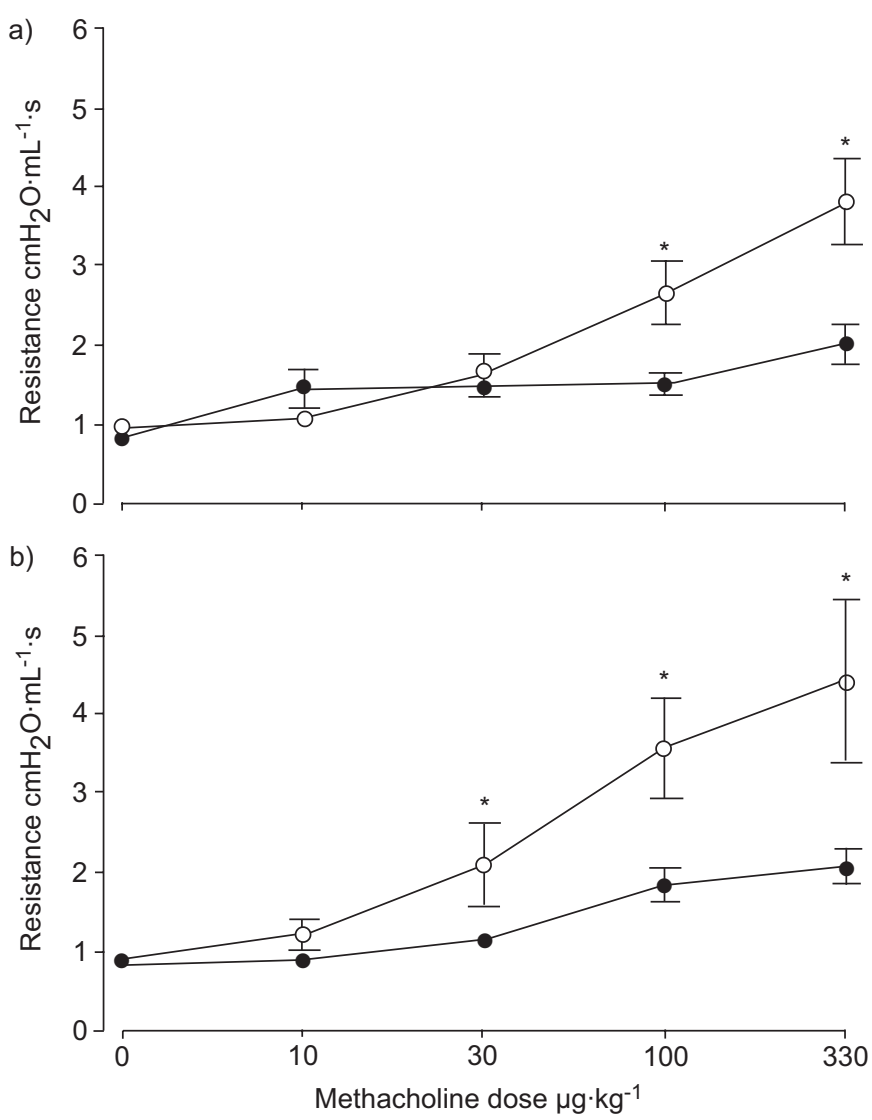

FIGURE 1. Physiology measurements in BALB/c mice during increasing doses of methacholine a) $24 \mathrm{~h}$ after brief exposure to saline $(\bullet)$ or ovalbumin (OVA; $O$ ) or b) 4 weeks after chronic exposure to saline $(\bullet)$ or OVA $(\bigcirc)$. Data are presented as mean \pm SEM. $n=10$ per group. ${ }^{*}: p<0.05$ compared with corresponding contro animals.

video rate even further to $0.1 \mathrm{fps}$ removes the oscillations, leaving only the moment-by-moment estimation of contraction velocity. Based on this analysis, $0.2 \mathrm{fps}$ was chosen as the optimal recording rate.

\section{Airway narrowing dynamics after brief or chronic allergen exposure}

In order to investigate whether the allergen-induced increased methacholine responsiveness was accompanied by greater velocity and/or magnitude of cholinergic-induced airway narrowing, lung slices were obtained from BALB/c allergenexposed animals and control animals. Acetylcholine was used at $1 \mu \mathrm{M}$ to assess responsiveness, this concentration has been shown to be essentially maximally effective $[11,15,16]$ and, as figure 1 did not indicate there to be a change in sensitivity, a full concentration-response relationship was unnecessary.

The maximal narrowing of the airways in response to $1 \mu \mathrm{M}$ acetylcholine did not differ significantly between BALB/c brief allergen-exposed mice and saline control mice (fig. 3a), nor did the peak velocity of that narrowing differ (fig. 3b). Similarly, after chronic allergen exposure, neither the maximal narrowing of the airways (fig. 3c) nor the velocity of that narrowing (fig. $3 \mathrm{~d}$ ) in response to $1 \mu \mathrm{M}$ acetylcholine were significantly different in allergen-exposed and saline control BALB/c mice.
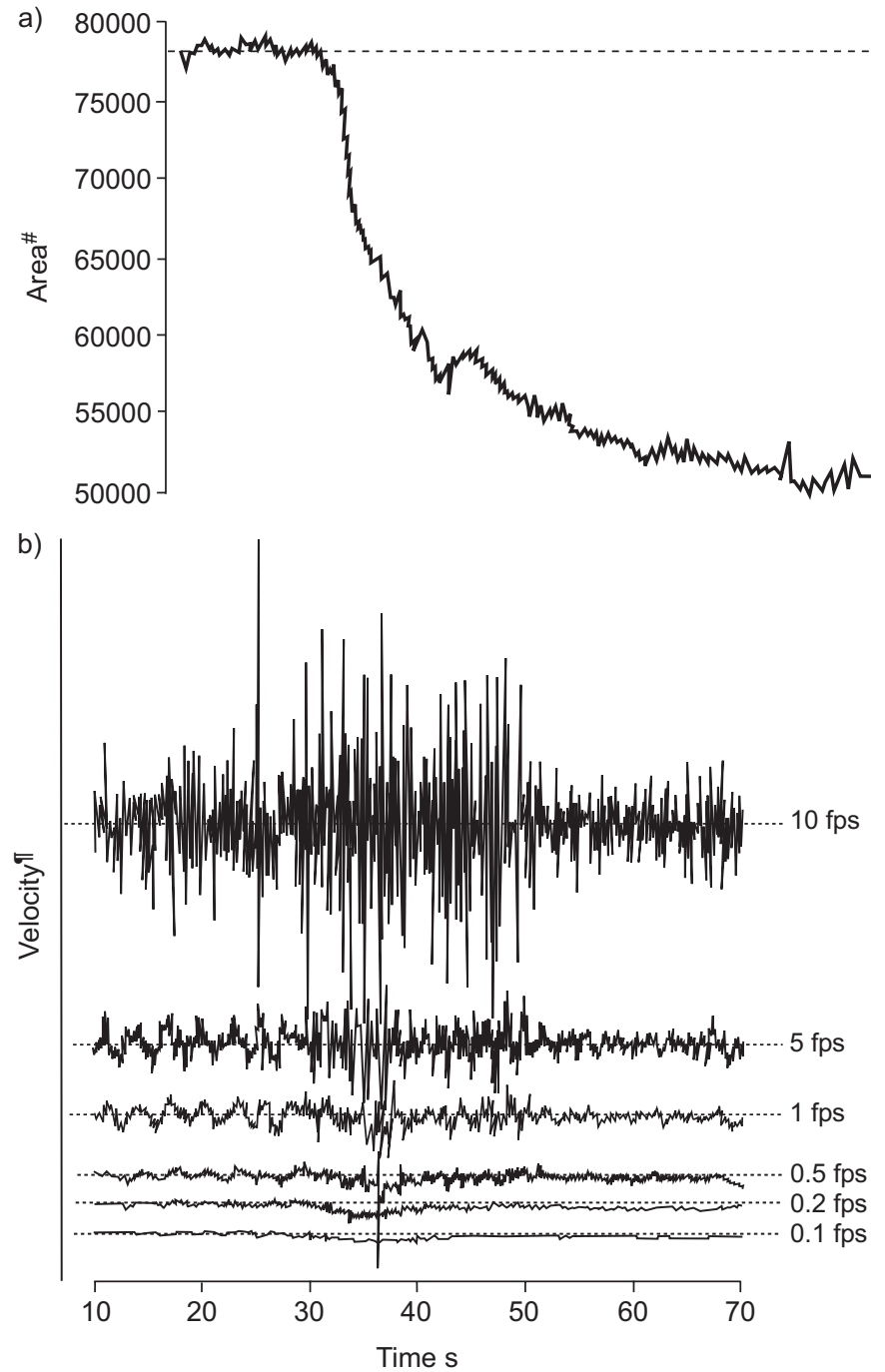

FIGURE 2. Effect of rate of data acquisition (video frame rate) on the estimation of velocity of airway narrowing. a) The response to acetylcholine $\left(10^{-5} \mathrm{M}\right)$ was recorded at 10 frames per second (fps). b) A 120-s segment of this recording beginning $\sim 30 \mathrm{~s}$ prior to onset of cholinergic stimulation, was extracted and reduced to different frame rates, which were subsequently analysed for velocity of airway narrowing. ${ }^{*}$ : measured as number of pixels; ${ }^{\bullet}: 1,000$ pixels $\cdot \mathbf{s}^{-1}$.

\section{Allergen-induced changes in airway wall structure}

In order to examine indices of airway remodelling in BALB/C mice following chronic allergen exposure, paraffin-embedded sections were stained and assessed by morphometric analysis as described previously [13]. There were significant increases in the amount of mucin-containing, periodic acid-Schiff positive goblet cells (fig. 4a), subepithelial collagen deposition (fig. $4 \mathrm{~b}$ ), and $\alpha$-SMA staining (fig. $4 \mathrm{c}$ ) in the airways of chronic allergen-exposed mice, compared with saline control mice $(\mathrm{p}<0.05)$.

\section{DISCUSSION}

Using two established models of allergen-induced AHR, the present authors provide evidence that allergen-induced increased responsiveness to methacholine assessed in vivo is not associated with increased airway narrowing velocity or 

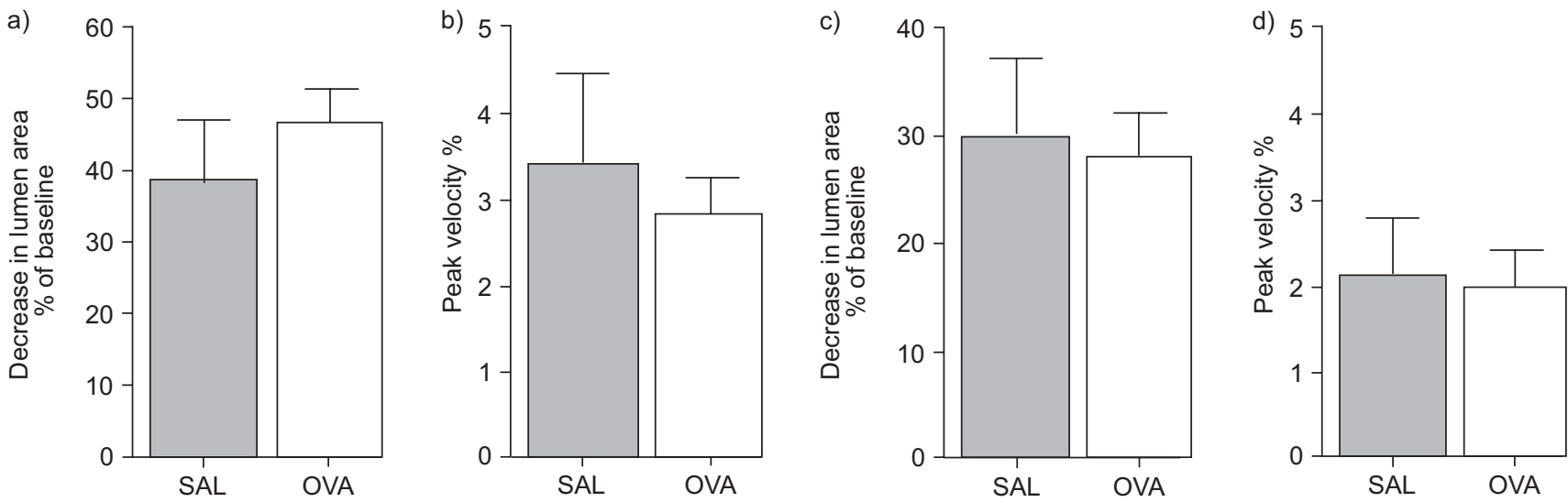

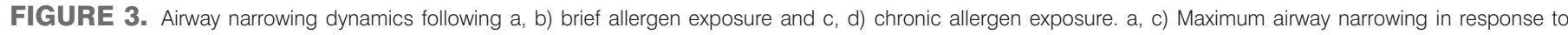

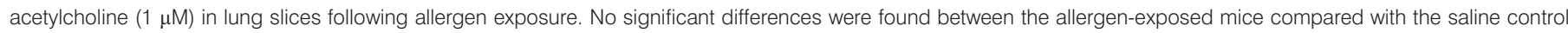

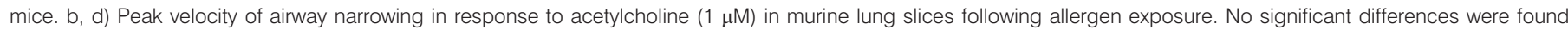

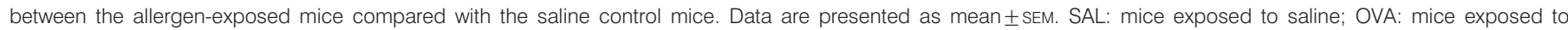
ovalbumin.

magnitude of narrowing measured in isolated lung tissues using an ex vivo lung-slice technique.

In recent years, experimental models of allergen exposure have been used in the study of asthma and the pathophysiological changes associated with it. The present authors' laboratory had characterised a murine model of brief allergen exposure resulting in transient AHR [12]. Although this model has been investigated for its airway responsiveness, airway inflammation via bronchoalveolar lavage (BAL) and tissue sections, to the authors' knowledge there are no data addressing whether or not airway contraction dynamics are altered following brief allergen exposure.

The present results demonstrate that neither maximum narrowing nor peak velocity differed between allergenexposed mice and saline control animals. The finding that no differences were detected between groups was surprising, given that an increase in BAL type-2 T-helper cytokines IL-4, -5 and -13 have been previously reported in this and other brief models of allergen exposure [12, 17, 18]. Those previous findings led to the hypothesis that the inflammatory mediators present in the airways could affect the dynamic properties of the airway contraction, thus providing a possible mechanism to explain the in vivo AHR observed in the model. Indeed, it is known that in vitro exposure of airway smooth muscle to IL-13 can increase specific (carbachol) and nonspecific $(\mathrm{KCl})$ contractions [4]. In the present study, it is possible that any inflammatory mediators that were present in vivo, are easily lost during the overnight incubation, as well as by superfusion during the experiment itself. Further experiments are warranted to address whether altered levels of cytokines are present in the lung slice tissue prior to and following allergen challenge. Another limitation of the present study is that relaxant responses were not compared in these two groups of animals.

In addition to the brief allergen exposure model described, chronic allergen exposure of the actively sensitised BALB/c mouse is another well-established model that includes several pathological features associated with the asthmatic airway. In the present model of chronic exposure to allergen, AHR persists for $\geqslant 8$ weeks following the final allergen exposure, well beyond the resolution of acute inflammatory events [14]. Structural changes in the airway, often termed airway remodelling, may be partly responsible for the sustained AHR in vivo. The present hypothesis was that these changes would also become manifest in airway dynamics in lung slices in vitro. In the present study, increased indices of airway remodelling in mice were observed 4 weeks after chronic allergen exposure. Specifically, increased deposition of subepithelial collagen, increased contractile tissue and goblet cell metaplasia were observed in the first generation airway in the left lobe. The present findings are consistent with previous studies by ELLIS et al. [14] and with other reports in the literature [17, 19]. Interestingly, following chronic allergen exposure, a significant increase in either maximum narrowing or peak velocity was not observed, despite the fact that BALB/ $\mathrm{C}$ mice had demonstrated increased indices of airway remodelling. A possible explanation could be that the intranasal allergen delivery system may not distribute the allergen to the small airways $(>200 \mu \mathrm{m}$ in diameter) used in the in vitro studies. However, the present authors have previously shown that structural changes are present in the first generation airway of the left lobe, as well as the smaller airways of the right lobe, suggesting that these changes are found throughout the entire bronchial tree [14]. Other models of chronic allergen have not determined the extent of structural changes in the lower airways [17].

An alternative explanation for the negative findings with ex vivo assessment of airway function could be that the small airways used to examine allergen-induced changes in maximal narrowing and velocity may not contribute to airway dysfunction assessed in vivo, despite having exhibited structural changes from repeated exposure to allergen. Indeed, the allergeninduced AHR observed in vivo may be indicative of changes in resistance from the larger airways as opposed to the smaller airways. It has been noted previously by SAPIENZA et al. [20] 

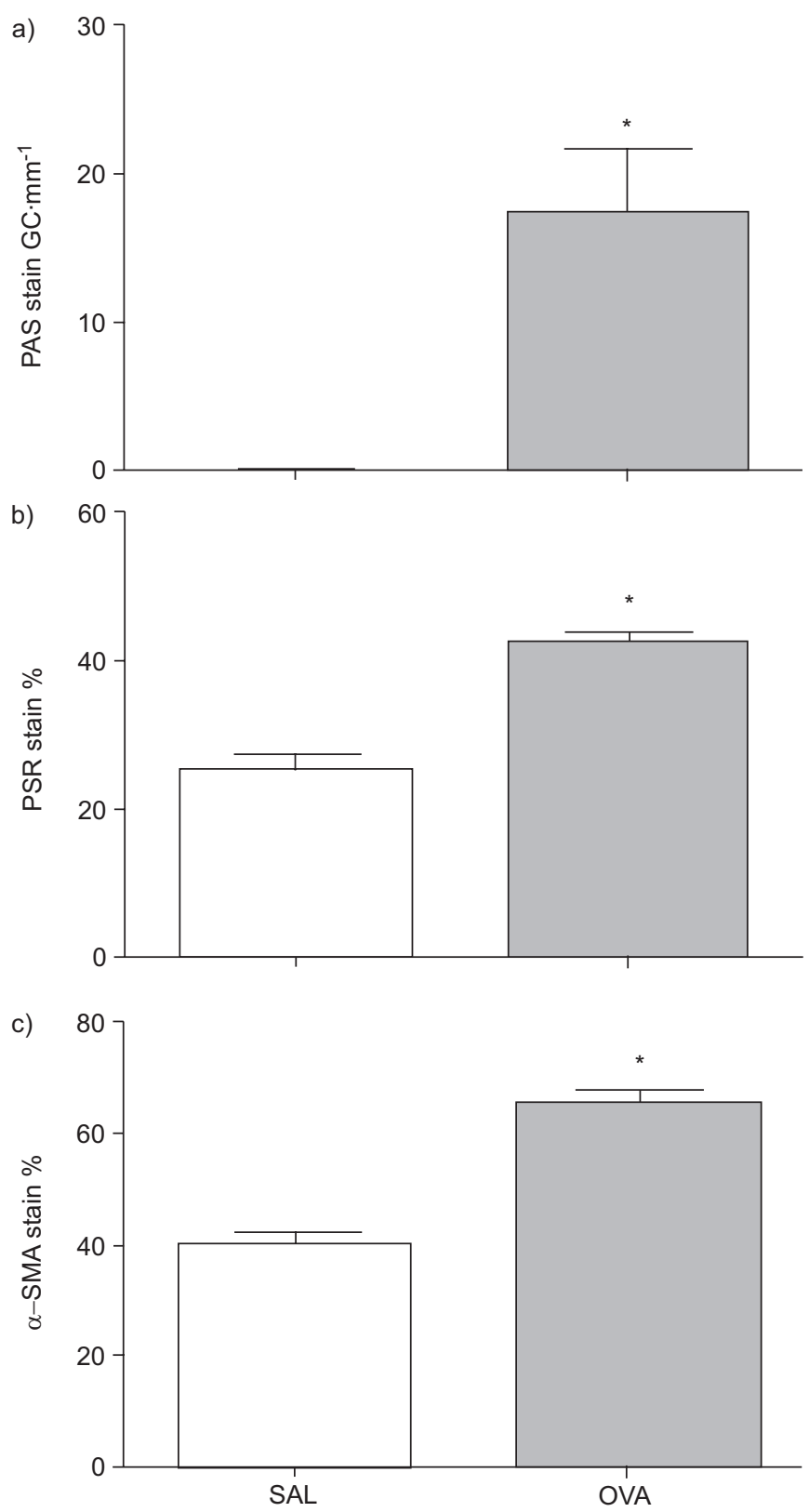

FIGURE 4. Morphometric changes in airways of mice following chronic exposure to saline (SAL) or ovalbumin allergen (OVA). Staining as assessed using morphometry for: a) mucin-containing, periodic acid-Schiff (PAS) positive goblet cells (GC) per millimetre basement membrane length in the epithelium measured using PAS stain; b) collagen measured as percentage Picrosirius red (PSR)stained; and c) contractile elements measured as percentage $\alpha$-smooth muscle actin (SMA) stained in the $20 \mu \mathrm{m}$ region beneath the epithelium. Data are presented as mean \pm SEM. * : $p<0.05$ compared with corresponding control animals.

that changes in pulmonary resistance after methacholine inhalation in rats were largely due to changes in the large airway level, not the small airways. Therefore, the reported AHR in mice exposed to allergen in the present authors' laboratory may be indicative of resistance changes in the large airways as well, although this hypothesis has not yet been tested. A third possibility is that the increased collagen deposition may be protective against exaggerated airway narrowing. This theory is supported by observations reported by PALMANS et al. [21] in a rat model of prolonged allergen exposure. In that study, an increase in collagen deposition accompanied by a decrease in AHR was observed after 4 weeks of continuous allergen exposure followed by a further increase after 12 weeks exposure. PALMANS et al. [21] reasoned that the increase in collagen could stiffen the airway wall, thus reducing the amount of airway narrowing for any given amount of ASM shortening. Furthermore, increased collagen deposited in and around the smooth muscle may also interfere with smooth muscle contraction and, subsequently, lead to a decrease in AHR [22].

In the present study, the in vivo measurement of responsiveness to methacholine is that of the entire respiratory system, which may reflect compartments other than the airway lumen diameter, including lung parenchyma. As such, the term airway responsiveness applied to these measurements may be inaccurate, as nonairway events may play a significant role. Therefore, it is possible that one of the reasons for any observed agreement between in vivo and ex vivo responsiveness changes after allergen exposure, was that a significant component of the allergen-induced change for the in vivo measurement was mediated through factors other than true changes in airway diameter. These may include changes in lung parenchymal resistance [23] or increased heterogeneity of airway diameter [24], without actual changes in airway resistance. While the present authors have not performed detailed analyses of precisely where the increase in methacholine induced RRS occurs after allergen, the data presented herein may indicate that the increased responsiveness to methacholine may not simply be due to increased narrowing of the airway.

In conclusion, an ex vivo lung-slice technique to assess cholinergic-induced airway contraction dynamics following allergen exposure was used. Although increased responsiveness to methacholine was detected in vivo, no differences in airways contraction dynamics could be shown following brief or chronic exposure to allergen within the BALB/c mouse strain. Airway hyperresponsiveness observed in mice in vivo is likely to be a result of factors other than intrinsic changes in smooth muscle dynamics and reflects changes in airways other than those observed using the present thin lung-slice technique.

\section{ACKNOWLEDGEMENTS}

The present authors would like to thank M. Sanderson and Y. Bai (Dept of Physiology, University of Massachusetts Medical School, Worcester, MA, USA) for their help with the lung-slice technique.

\section{REFERENCES}

1 O'Byrne PM, Inman MD. Airway hyperresponsiveness. Chest 2003; 123: Suppl. 3, 411S-416S.

2 Kuperman DA, Huang X, Koth LL, et al. Direct effects of interleukin-13 on epithelial cells cause airway hyperreactivity and mucus overproduction in asthma. Nat Med 2002; 8: 885-889.

3 Wills-Karp M, Luyimbazi J, Xu X, et al. Interleukin-13: central mediator of allergic asthma. Science 1998; 282: 2258-2261. 
4 Tliba O, Deshpande D, Chen $\mathrm{H}$, et al. IL-13 enhances agonist-evoked calcium signals and contractile responses in airway smooth muscle. Br J Pharmacol 2003; 140: 1159-1162.

5 Antonissen LA, Mitchell RW, Kroeger EA, Kepron W, Tse KS, Stephens NL. Mechanical alterations of airway smooth muscle in a canine asthmatic model. J Appl Physiol 1979; 46: 681-687.

6 Duguet A, Biyah K, Minshall E, et al. Bronchial responsiveness among inbred mouse strains. Role of airway smooth-muscle shortening velocity. Am J Respir Crit Care Med 2000; 161: 839-848.

7 Fan T, Yang M, Halayko A, Mohapatra SS, Stephens NL. Airway responsiveness in two inbred strains of mouse disparate in IgE and IL-4 production. Am J Respir Cell Mol Biol 1997; 17: 156-163.

8 Ma X, Cheng Z, Kong H, et al. Changes in biophysical and biochemical properties of single bronchial smooth muscle cells from asthmatic subjects. Am J Physiol Lung Cell Mol Physiol 2002; 283: L1181-L1189.

9 Stephens NL, Li W, Wang Y, Ma X. The contractile apparatus of airway smooth muscle. Biophysics and biochemistry. Am J Respir Crit Care Med 1998; 158: S80-S94.

10 Wang CG, Almirall JJ, Dolman CS, Dandurand RJ, Eidelman DH. In vitro bronchial responsiveness in two highly inbred rat strains. J Appl Physiol 1997; 82: 1445-1452.

11 Bergner A, Sanderson MJ. Airway contractility and smooth muscle $\mathrm{Ca}^{2+}$ signaling in lung slices from different mouse strains. J Appl Physiol 2003; 95: 1325-1332.

12 Leigh R, Ellis R, Wattie J, et al. Dysfunction and remodeling of the mouse airway persist after resolution of acute allergen-induced airway inflammation. Am J Respir Cell Mol Biol 2002; 27: 526-535.

13 Bergner A, Sanderson MJ. Acetylcholine-induced calcium signaling and contraction of airway smooth muscle cells in lung slices. J Gen Physiol 2002; 119: 187-198.
14 Ellis R, Leigh R, Southam D, O'Byrne PM, Inman MD. Morphometric analysis of mouse airways after chronic allergen challenge. Lab Invest 2003; 83: 1285-1291.

15 Khan M, Kianpour S, Stämpfli M, Janssen LJ. Kinetics of in vitro bronchoconstriction in an elastolytic mouse model of emphysema. Eur Respir J 2007; 30: 691-700.

16 Bai Y, Zhang M, Sanderson MJ. Contractility and $\mathrm{Ca}^{2+}$ signaling of smooth muscle cells in different generations of mouse airways. Am J Respir Cell Mol Biol 2007; 36: 122-130.

17 McMillan SJ, Lloyd CM. Prolonged allergen challenge in mice leads to persistent airway remodelling. Clin Exp Allergy 2004; 34: 497-507.

18 Corry DB, Folkesson HG, Warnock ML, et al. Interleukin 4, but not interleukin 5 or eosinophils, is required in a murine model of acute airway hyperreactivity. J Exp Med 1996; 183: 109-117.

19 Temelkovski J, Hogan SP, Shepherd DP, Foster PS, Kumar RK. An improved murine model of asthma: selective airway inflammation, epithelial lesions and increased methacholine responsiveness following chronic exposure to aerosolised allergen. Thorax 1998; 53: 849-856.

20 Sapienza S, Du T, Eidelman DH, Wang NS, Martin JG. Structural changes in the airways of sensitized brown Norway rats after antigen challenge. Am Rev Respir Dis 1991; 144: 423-427.

21 Palmans E, Kips JC, Pauwels RA. Prolonged allergen exposure induces structural airway changes in sensitized rats. Am J Respir Crit Care Med 2000; 161: 627-635.

22 McParland BE, Macklem PT, Pare PD. Airway wall remodeling: friend or foe? J Appl Physiol 2003; 95: 426-434.

23 Romero PV, Zin WA, Lopez-Aguilar J. Frequency characteristics of lung tissue strip during passive stretch and induced pneumoconstriction. J Appl Physiol 2001; 91: 882-890.

24 Peták F, Hantos Z, Adamicza A, Asztalos T, Sly PD. Methacholine-induced bronchoconstriction in rats: effects of intravenous versus aerosol delivery. J Appl Physiol 1997; 82: 1479-1487. 